\title{
IN MEMORIAM: CZESŁAW RYLL-NARDZEWSKI'S CONTRIBUTIONS TO PROBABILITY THEORY
}

\author{
BY
}

TOMASZ ROLSKI (WROCŁAW) AND WOJBOR A. WOY CZY ŃS K I (CLEVELAND)

\begin{abstract}
In this paper we review contributions of late Czesław RyllNardzewski to probability theory. In particular, we discuss his papers on point processes, random power series, random series in infinite-dimensional spaces, ergodic theory, de Finetti's exchangeable sequences, conditional distributions and applications of the Kuratowski-Ryll-Nardzewski theorem on selectors.
\end{abstract}

2010 AMS Mathematics Subject Classification: Primary: 01Axx; Secondary: 60-03.

Key words and phrases: Ryll-Nardzewski, biography, probability theory.

\section{A BIOGRAPHICAL SKETCH}

Czesław Ryll-Nardzewski, commonly and affectionately referred to as CRN during his time in Wrocław (we will preserve this moniker in this article), was born on October 7, 1926, in Wilno, then in Polish Lithuania, and died on September 18, 2015, in Wrocław, Poland, the city where he spent almost all of the last 65 years of his life. In 1948 he graduated from the Marie Curie-Skłodowska University in Lublin, and in 1949 defended his PhD dissertation Distribution Theory and $B_{0}$-Spaces written under the supervision of Mieczysław Biernacki. Shortly thereafter he took a position at the University and Politechnic of Wrocław. When the two schools separated in 1951, he settled at the University of Wrocław where, following a brief stint at Warsaw University, he was promoted to full professorship in 1964. In 1976 he moved across the street to the Wrocław University of Technology from which he retired in 2005. Since 1950 he has also held a secondary position at the Mathematical Institute of the Polish Academy of Sciences; he was first elected to membership of the Academy in 1967. Among his many activities in the mathematical community we would like to mention his service on the editorial boards of Studia Mathematica and Colloquium Mathematicum, and his significant contribution as a co-founding editor of Probability and Mathematical Statistics. He 


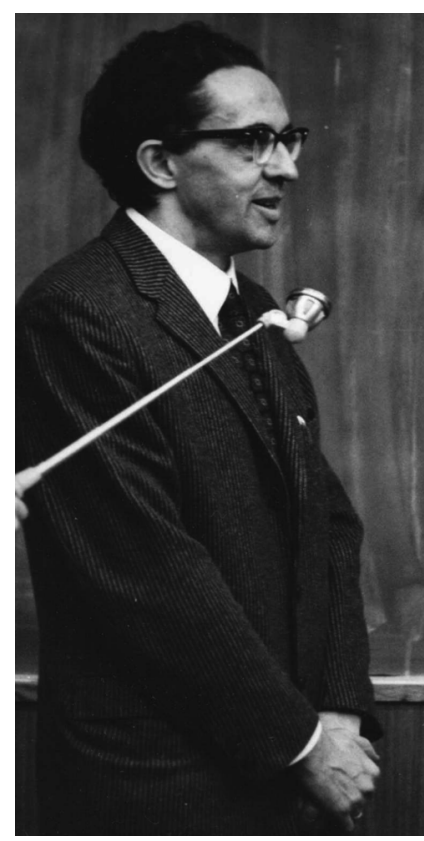

also served a term (1964-1966) as Dean of the Faculty of Mathematics, Physics and Chemistry at the University of Wrocław.

One of the most universal and versatile mathematicians in postwar Poland, he was legendary for his original insights and elegance of his ideas, proofs, and discoveries. His research ranged over many fields of mathematics, from model theory, measure theory, probability, topology, to functional and harmonic analysis, and foundations of mathematics. In this article we review only his work on probability theory including topics in point processes, de Finetti sequences, random functional series and ergodic theorems. These superb contributions introduced novel ideas and techniques that have stimulated new devel-

Czesław Ryll-Nardzewski in the 1970s.

opments. A list of selected CRN's papers on probability theory is enclosed in References. It is only a part of his full set of publications, many of which constitute outstanding and often pivotal contributions to different areas of mathematics.

In Wrocław, in the 1950s, it was Hugo Steinhaus' and, partly, Edward Marczewski's influences that attracted CRN to probabilistic topics. The years from 1951 to 1953 were truly anni mirabiles for the young mathematician. Volume 12 (1951) of Studia Mathematica contains the following eight papers by CRN and his coauthors :

- Sur les produit de composition (with J. Mikusiński),

- On the ergodic theorems. I (Generalized ergodic theorems),

- On the ergodic theorems. II (Ergodic theory of continued fractions),

- Sur les fonctions indépendantes. IX (Séries des fonctions positives) (with H. Steinhaus),

- Sur les suites et les fonctions également réparties,

- Sur les séries de Taylor (with H. Steinhaus),

- Sur l'opérateur de translation (with J. Mikusiński),

- Certaines théorèmes des moments.

And this feat was then continued in the next volume, 13 (1953), with the appearance of another series of seven articles (the details are listed in the bibliography, see [1]-[15]),

- D. Blackwell's conjecture on power series with random coefficients, 
- Sur la convergence des séries de puissances de l'opérateur différentiel,

- Sur les séries de puissances dans le calcul opératoire,

- A theorem on bounded moments (with J. Mikusiński),

- Un théorème sur le produit de composition des fonctions de plusieurs variables (with J. Mikusiński),

- Remarks on the Poisson stochastic process. I (with K. Florek and E. Marczewski),

- Théorèmes abstraits de Kronecker et les fonctions presque périodiques (with S. Hartman).

In what follows we describe the main contributions of Czesław Ryll-Nardzewski to probability theory and related fields, and discuss their influence on the work of other mathematicians.

\section{POINT PROCESSES AND RANDOM MEASURES}

2.1. Poisson processes. Let us begin by recalling what is currently meant by a Poisson process on $(E, \mathcal{E})$, where (like in the book by Daley and Vere-Jones [34], p. 34) $E$ is a complete separable metric space, and $\mathcal{E}$ the Borel $\sigma$-field generated by open balls of $E$. Denote by $\mathcal{N}$ the suitably topologized space of boundedly finite counting measures, that is, measures taking values in the set of nonnegative integers complemented by $+\infty$, which take finite values on bounded sets.

Definition. A measurable mapping $N:(\Omega, \mathcal{F}, \mathbb{P}) \rightarrow(\mathcal{N}, \mathcal{B}(\mathcal{N}))$ is said to be a point process. A point process is said to be a Poisson point process with boundedly finite parameter measure $m$ if

(PP1) for any family of disjoint bounded sets, $B_{j} \in \mathcal{E}(j=1, \ldots, m)$, the random variables $N\left(B_{1}\right), \ldots, N\left(B_{m}\right)$ are independent, and

(PP2) for every $B \in \mathcal{E}, N(B)$ is a Poisson random variable with mean $m(B)$.

The term "point processes" appeared for the first time in the 1940s, when William Feller used it in a 1940 paper, and Ove Lundberg, and Conny Palm, employed it in their 1940, and 1943, respectively, PhD dissertations. For Palm, who wrote in German, the term was "Punktprozesse". For simple point processes, condition (PP2) alone defines a Poisson process.

In the 1950s, in Wrocław, it was Edward Marczewski (see [15]), in collaboration with Kazimierz Florek and CRN, who initiated a thorough study of Poisson processes. In their work the space $E=[0, \infty)$, and the Poisson process $\{N(t)\}$ was an integer-valued, right-continuous, and non-decreasing stochastic process with independent and stationary increments. Today, such a process is often called the counting process, and the increments $N(t+s)-N(t)$ are interpreted as a number of random points in the interval $(t, t+s]$. In another paper from that period Marczewski (see [52]), starting with the concept of the counting process $N(t)$, was able to extend the notion to the number of points $N(B)$ in a Borel set $B$ (see also Cramér [33]). 
Then, in a pioneering 1954 Studia Mathematica paper, [17], Ryll-Nardzewski introduced the notion of a non-homogeneous Poisson point process in the form that is commonly used today. He considered a fixed Borel subset $E$ of a finitedimensional Euclidean space and a countable field of its Borel subsets $\mathcal{E}_{0}$ generating the $\sigma$-field $\mathcal{E}$ of Borel subsets of $E$. Then, the probability space $\Omega$ was taken to be the space of finite, real-valued set functions on $\mathcal{E}_{0}$ which are $\sigma$-additive, with a probability measure $\mathbb{P}$ defined on a $\sigma$-field of subsets of $\Omega$. His assumptions were as follows:

(1) $\mathcal{E}_{0} \ni B \mapsto \omega(B)$ is $\mathbb{P}$-measurable, and

(2) $\omega\left(B_{1}\right), \ldots, \omega\left(B_{k}\right)$ are independent, whenever $B_{j}$ are disjoint sets belonging to $\mathcal{E}_{0}$.

The paper called a point $x_{0} \in E$ singular if

$$
\mathbb{P}\left(\omega\left(\left\{x_{0}\right\}\right) \neq 0\right)>0,
$$

and defined what today is called the intensity measure, $m(B)=\int \omega(B) d \mathbb{P}$. The key assumption was that the point process is simple, that is, $\mathbb{P}\left(\Omega_{1}\right)=1$, with $\Omega_{1} \subset \Omega$ consisting only of purely atomic set functions such that $\omega\left(x_{0}\right)=1$ for every atom $x_{0}$. The main result was the following

THEOREM. Suppose that $m$ is a finite measure on $\mathcal{E}$. If $x_{0} \in E$ is an atom of $m$ (or, in other words, if $x_{0}$ is singular), then $\omega\left(\left\{x_{0}\right\}\right)$ assumes the value 1 , or 0 , with probability $m\left(\left\{x_{0}\right\}\right)$, or $1-m\left(\left\{x_{0}\right\}\right)$, respectively. If $B$ contains no singular points, then $\omega(B)$ has the Poisson distribution

$$
\mathbb{P}(\omega(B)=k)=\frac{m(B)^{k}}{k !} e^{-m(B)} .
$$

The remainder of the paper extended the theorem to the case when $m$ is $\sigma$ finite.

In his 1954 paper, [18], continuing the series of papers on Poisson processes, CRN defined the point process by starting with a random countable set $\boldsymbol{X}$ of real numbers, defined by a sequence $\left\{X_{j}\right\}$ of random variables. Then, for each interval $I \subset \mathbb{R}$ he introduced the random variable

$$
N(I)=\text { the number of indices } j \text { such that } X_{j} \in I,
$$

and made the following assumptions:

(i) $\mathbb{P}\left(X_{j} \neq X_{k}\right)=1$ for $j \neq k$,

(ii) $\mathbb{P}\left(\lim _{j}\left|X_{j}\right|=\infty\right)=1$, and

(iii) $\mathbb{P}\left(X_{j}=a\right)=0$ for every $j$, and real $a$.

If, for any disjoint intervals, $I_{1}, \ldots, I_{k}$, random variables $N\left(I_{1}\right), \ldots, N\left(I_{k}\right)$ are statistically independent, then it follows from an earlier CRN's paper, [17], that the distribution $\mathbb{P}(N(I)=k)$ is Poissonian, that is, for some $\sigma$-finite measure $m$ on 
the line, equation (2.11) is satisfied. In [118], the special case of the homogeneous (stationary) processes, with $m(I)=\lambda|I|$, was considered. It is interesting to note that in the above paper CRN was probably the first who discovered what Kingman [47] later called a characterization of Poisson processes by Bernoulli processes:

LEMMA. For a given interval $I \subset \mathbb{R}$, the conditional probability distribution, given $N(I)=k$, of the set of $k$ points of $\boldsymbol{X}$ belonging to $I$ is the same as the uniform distribution of $k$ independent points belonging to $I$.

The paper also introduced the following transform,

$$
\phi(f)=\mathbb{E} \prod_{j}\left(1+f\left(X_{j}\right)\right),
$$

for a complex-valued function $f$ of a real variable such that $\int_{-\infty}^{\infty}|f(t)| d t<\infty$. The transform could be interpreted as a "characteristic functional". Under the assumption that $\boldsymbol{X}$ is a homogeneous Poisson process, employing the above Lemma, he was able to prove that this characteristic functional is of the form

$$
\phi(f)=\exp \left(\lambda \int_{-\infty}^{\infty} f(t) d t\right) .
$$

This result should be compared with more general theorems on probability generating functionals defined, for suitable test functions $h$ (see [34], p. 15), by the expression $\mathbb{E} \prod_{j} h\left(x_{j}\right)$, or results on the Laplace functional contained in Olav Kallenberg's book on random measures (see [42]).

Having established the tool described in the above Lemma, CRN was ready to prove the main theorem of the paper:

THEOREM. If $\left\{X_{j}\right\}$ is a homogeneous Poisson process, and $\left\{Y_{j}\right\}$ is an independent sequence of exchangeable random variables, then the process with points $\left\{X_{j}+Y_{j}\right\}$ is again a homogeneous Poisson process, with the same parameter $\lambda$ as $\left\{X_{j}\right\}$.

Recall that a sequence $\left\{Y_{i}\right\}$ of random variables is said to be exchangeable (in the sense of de Finetti) if, for any $k$ and any permutation $i_{1}, i_{2}, \ldots, i_{k}$ of the indices $1,2, \ldots, k$, the joint distribution of $Y_{i_{1}}, Y_{i_{2}}, \ldots, Y_{i_{k}}$ is independent of the permutation. We will return to this notion in Section 5.

2.2. A new approach to point processes. In their 2003 monograph on point processes, [34], Daley and Vere-Jones wrote that Ryll-Nardzewski's 1961 Berkeley Symposium contribution on processes of calls, [20], set out fundamental properties of point processes, and provided a new and more general approach to the so-called Palm probabilities. In the introduction to the Berkeley paper Ryll-Nardzewski wrote: "The theory of processes of calls is highly developed. In this paper I am going to consider some questions which, to my mind, have not yet been analyzed 
sufficiently from the measure theoretic point of view." Then he cited the work of two of his predecessors in the field: C. Palm and A. Ya. Khinchin. In particular, for simple stationary point processes $\mathrm{CRN}$ gives a relationship between two probability measures: stationary and Palm. A particular case appeared earlier, known as the Palm-Khinchin equation, but it was a relationship between two functions (see [44]). In the case of renewal theory, the formula illuminates the so-called Feller's waiting time paradox (see [37], p. 12).

The paper clarified and completed his earlier ideas on point processes reviewed in the previous subsection. Here, $\Omega$ was the class of all countable subsets of the real line, $\mathbb{R}$, which have a finite intersection with each bounded interval. For each $\omega \in \Omega$, he defined

$$
N(\omega, B):=\operatorname{card}(\omega \cap B),
$$

and selected as the class of measurable subsets $\mathcal{F}$ the $\sigma$-field generated by all the functions $N(\cdot, B)$, where $B$ is a Borel set. He observed that each $N$ can also be treated as a purely atomic measure, finite for bounded sets. This established the modern approach to point processes, where by a point process one means a measurable mapping to the space of atomic measures $\mathcal{N}$. The next important remark was that $(\Omega, \mathcal{F})$ can be one-to-one mapped to the unit interval $I=[0,1]$ and the class of all Borel subsets of $I$, which made it possible to avoid the difficulties related to regular versions of conditional probabilities.

One of the fundamental questions in the theory of point processes of that period was how to define the conditional probability of a point process under the condition of zero probability. Ryll-Nardzewski's approach to define what is today called Palm distributions was as follows: Assume that, for a bounded $B$, the mean measure satisfies the condition $m(B)=\mathbb{E} N(B)<\infty$, and for each event $A \in \mathcal{F}$, the integral

$$
\int_{A} N(\omega, B) \mathbb{P}(d \omega),
$$

treated as a function of the set $B$, is absolutely continuous with respect to $m$. Then, by the Radon-Nikodym theorem, we can write

$$
\int_{A} N(\omega, B) \mathbb{P}(d \omega)=\int_{B} \Pi(A \mid t) m(d t),
$$

where $\Pi(A \mid t)$ is an $m$-measurable function of $t$.

For each fixed $A$, the Radon-Nikodym derivative $\Pi(A \mid t)$ is unique $m$-a.e., and we can always assume that it is a true probability measure over all sets $A \in \mathcal{F}$. The formula (2.2) can be generalized to the following equation,

$$
\int f(\omega) N(\omega, B) \mathbb{P}(d \omega)=\int_{B} m(d t) \int f(\omega) \Pi(d \omega \mid t)
$$

for functions $f$ which are $\mathbb{P}$-integrable. The above arguments remain valid for an arbitrary complete separable metric space $E$, and they became a standard technique to define such conditional distributions for nonstationary point processes. 
For point processes on the real line, stationarity becomes an important issue. Let us recall that a point process is said to be stationary if, for all $t$ and $A$, we have $\mathbb{P}(A)=\mathbb{P}\left(A^{t}\right)$, where we define the shift by the formulas: $\omega^{t}=\omega+t$, and $\left\{\omega \in A^{t}\right\}=\left\{\omega^{-t} \in A\right\}$. Under the assumption $\mathbb{P}(N(\mathbb{R})=0)=0$, we obtain $m(d t)=\lambda d t$, where $\lambda$ is called the intensity of the point process. The following CRN's theorem established the existence of Palm distributions for such processes.

THEOREM. There exists one, and only one probability measure $\mathbb{P}^{o}$ defined on $(\Omega, \mathcal{F})$, for which the measure,

$$
\Pi(B \mid t)=\mathbb{P}^{o}\left(B^{-t}\right),
$$

depending on parameter $t$, satisfies the condition (2.2) for all $B$ and $A$.

In this situation, $(2.3)$ is a special form of the so-called Campbell-Mecke equation, ${ }^{1}$

$$
\int f(\omega) N(\omega, B) \mathbb{P}(d \omega)=\lambda \int \mathbb{P}^{o}(d \omega) \int_{B} f\left(\omega^{t}\right) d t .
$$

An important observation is that $\mathbb{P}^{o}\left(\Omega_{0}\right)=1$ for $\Omega_{0}=\{\omega: 0 \in \omega\}$. Then one can define random variables $\eta_{j}, j \in \mathbb{Z}$, on $\left(\Omega_{0}, \Omega_{0} \cap \mathcal{F}, \mathbb{P}^{o}\right)$ as distances between points. Suppose that $\eta_{0}$ is the distance from zero to the nearest point to the right. CRN's major result is given in the following theorem.

THEOREM. Under the above assumption we have

(i) $\eta_{j}>0$

(ii) $\mathbb{E}^{o} \eta_{j}=\lambda^{-1}$,

(iii) $\left\{\eta_{j}\right\}$ form a stationary sequence.

Conversely, each sequence of random variables $\left\{\eta_{j}\right\}$ satisfying (i)-(iii) can be obtained in this way. Moreover, the correspondence between $\mathbb{P}$ and $\mathbb{P}^{o}$ is one-toone, and it is given by the equation

$$
\int f(\omega) \mathbb{P}(d \omega)=\lambda \int_{\Omega_{0}} \mathbb{P}^{o}(\omega) \int_{0}^{\eta_{0}} f\left(\omega^{-t}\right) d t .
$$

2.3. The oldest individual and random measures on Boolean algebras. The above fundamental work of Ryll-Nardzewski influenced the next generation of probabilists. Kingman [46] writes: "The advantage of working directly in terms of random measures becomes more obvious when the space involved is more complicated than the real line. For example, a point process in several dimensions ... would be difficult to work with in terms of the analogue of $X(t)$ [the counting

${ }^{1}$ See formula (12.1.11) in [55], and also compare with [28], where it is called the CampbellMathes formula. 
process], but it seems easy and natural to consider a random measure $\Phi$ such that $\Phi(B)$ is the number of points of the process in the set $B$." Such an approach is implicit in the work of Ryll-Nardzewski $[20]^{2}$ on random measures, and Kallenberg [42] gives ample credit to CRN's influence on the theory.

CRN's work on random measures also had a critical influence on the next generation working in the area. Here, we shall give just two examples from our own work. In 1970, Domokos Szász and Wojbor A. Woyczyński [57] proved the following result:

THEOREM. If $\mathcal{B}$ is a Boolean $\sigma$-algebra, $\mathcal{N}$ denotes the space of nonnegative integer-valued random variables, and $X: \mathcal{B} \rightarrow \mathcal{N}$ is a random measure with independent values on disjoint elements, then, for every $b \in \mathcal{B}$, with probability one,

$$
X(b)=\sum_{a_{i} \leqslant b} X\left(a_{i}\right)+\sum_{m=1}^{\infty} m X^{(m)}(b),
$$

where $X^{(m)}, m=1,2, \ldots$, are Poissonian random measures on $\mathcal{B}$. Moreover, all random variables $X\left(a_{i}\right)$ (atoms) and mappings $X^{(m)}$ are mutually independent.

This result was also motivated by the work of Khinchin [45], Rényi [54], and Prékopa [53], but the proof technique was directly influenced by CRN's work. The authors thanked CRN personally for helping to improve the paper; as a member of the Polish Academy of Sciences, he also submitted it to the Academy Bulletin.

And, in 1987, Tomasz Rolski published the paper [27] (coauthored with CRN) which answered the intriguing question about probability of the death of the oldest individual in a population. The problem was modeled by a stationary Poisson process with intensity $\lambda$ on $\mathbb{R}$ which represented the birth instants. To all the points were attached the life times which formed a sequence of independent, identically distributed nonnegative random variables with distribution $G$, and independent of the Poisson process. Calling the oldest individual at time $t$ the senior, and denoting its age by $Y(t)$, the paper has shown that the probability of death of the oldest individual is

$$
p=\int_{0}^{\infty} e^{-\lambda \int_{t}^{\infty}(1-G(u) d u)} d G(t) .
$$

The paper also studied the senior process $Y(t)$, and the proofs employed the concept of a non-homogeneous Poisson process on $\mathbb{R} \times \mathbb{R}_{+}$.

\footnotetext{
${ }^{2}$ Although [20] only considered point processes defined on the real line, the setting was obviously applicable to the more general case of multidimensional point processes.
} 


\section{RANDOM SERIES}

3.1. Random power series and the natural boundary. CRN has contributed a number of problems to the New Scottish Book (see [29]) that aroused interest in the international mathematical community. Here, a good example (published in Colloquium Mathematicum) is

PROBLEM 194 [July 2, 1952, Czesław Ryll-Nardzewski (Wrocław)]. If the coefficients of the expansion of a complex function $f(x)=\sum_{n=-\infty}^{\infty} a_{n} e^{\text {int }}$ satisfy the condition $\sum_{n=-\infty}^{\infty}\left|a_{n}\right|<\infty$, is it true that $|f(x)|$ has the absolutely convergent Fourier series?

The answer turns out to be "No", and the result was proven by a French mathematician Jean-Pierre Kahane in [40]. The paper launched Kahane's distinguished career in the theory of random functional series that culminated in publication of his celebrated monograph [41].

The idea of studying random series of functions goes back to the 1896 Émile Borel's paper [3]], but the first significant step in the theory was made by Hugo Steinhaus who in 1930, in [56], proved the following result:

THEOREM. If $\left\{r_{n}\right\}$ is a sequence of positive numbers satisfying the condition

$$
0<\lim \sup r_{n}^{1 / n}<\infty,
$$

and $\omega_{n}$ are independent random variables uniformly distributed on $[0,1]$, then the function

$$
F(z)=\sum_{n=0}^{\infty} r_{n} e^{2 \pi i \omega_{n}} z^{n}
$$

has, with probability one, its circle of convergence as a natural boundary.

Recall that if a power series has the radius of convergence $r$, and defines an analytic function $f$ inside the disc of that radius, then a point on the circle of convergence for which there is a neighborhood on which $f$ has an analytic extension is called regular, otherwise it is called singular. The circle is called a natural boundary (of analyticity) if all its points are singular. A 1932 paper by Paley and Zygmund extended Steinhaus' result to random series of the form

$$
F(z)=\sum_{n=0}^{\infty} \pm r_{n} z^{n}
$$

where \pm form the Rademacher sequence.

In 1947, in an oral communication to Hugo Steinhaus, David Blackwell conjectured that a random series

$$
\sum_{n=0}^{\infty} X_{n} z^{n}
$$


with independent coefficients $X_{n}$, can either have the circle of convergence as a natural boundary, or one can add a deterministic Taylor series to it so that the resulting random Taylor series has a strictly larger circle of convergence which is its natural boundary. This is the case in the simple example of the random series

$$
F(z)=\sum_{n=0}^{\infty}\left(2^{n} \pm 1\right) z^{n}
$$

which has the radius of convergence equal to $1 / 2$. But, when one subtracts the deterministic Taylor series $\sum_{n=0}^{\infty} 2^{n} z^{n}$ from $F(z)$, the remaining power series, with random independent symmetric Bernoulli coefficients \pm 1 , has the radius of convergence equal to one in view of the classical Kolmogorov's inequality, see [50]. In the 1953 paper, [10], CRN proved that Blackwell's guess was correct. More precisely, the Ryll-Nardzewski theorem (widely quoted and used in many papers and monographs, e.g., Kahane [41]) can be stated as follows:

THEOREM. Given $r_{F}>0$, either $|z|=r_{F}$ is a.s. a natural boundary for $F(z)=\sum_{n=0}^{\infty} X_{n} z^{n}$, or there exists a deterministic function $f(z)=\sum_{n=0}^{\infty} a_{n} z^{n}$ such that $|z|=r_{F-f}>r_{F}$ is a.s. a natural boundary for $F(z)-f(z)$.

The ingenious method of proof introduced by CRN was then used to demonstrate several other results in the area including the analogous result for random Dirichlet series

$$
\sum_{n=0}^{\infty} X_{n} e^{-\lambda_{n} s}
$$

where $\lambda_{0}>\lambda_{1}>\ldots>0, X_{n}$ are independent complex-valued random variables, and $s$ is complex, and to prove the Cartan-Thullen theorem stating that a polynomially convex open set is a domain of holomorphy. Recall that an open set $H$ in $\mathbf{C}^{m}$ is called polynomially convex if, given any point $\left(a_{1}, \ldots, a_{m}\right)$ in the exterior of $H$, there exists a polynomial $P\left(z_{1}, \ldots, z_{m}\right)$ such that $|P|<1$ on $H$, and $\left|P\left(a_{1}, \ldots, a_{m}\right)\right|>1$ (see, e.g., [41]], p. 37).

3.2. Convergence of vector random series in infinite-dimensional spaces. In the middle of 1970s, in a series of papers [23], [25], [24] (see also the Appendix in [50], which contains a systematic exposition of all the results described in this subsection) CRN and his collaborators have returned to the problems involving random series, but this time in the context of random variables with values in infinite-dimensional spaces, such as Banach spaces. The results are summarized below.

Consider a series $\sum_{k=1}^{\infty} X_{k}$ of random variables $X_{1}, X_{2}, \ldots$, defined on a probability space $(\Omega, \mathcal{F}, P)$, and with values in a Banach space $\mathbf{F}$. The following facts are well known and elementary:

PROPOSITION. If $\sum X_{k}$ is considered as a series in a complete metric linear space $\mathbf{E}$ (say, a subspace of $L_{0}(\Omega, \mathcal{F}, P ; \mathbf{F})$ ), then the following conditions are 
equivalent:

(i) the series $\sum X_{k}$ converges unconditionally, i.e., the series converges in $\mathbf{E}$ after arbitrary permutation of its terms;

(ii) all the series $\sum X_{k_{i}}$ of subsequences $X_{k_{1}}, X_{k_{2}}, \ldots\left(k_{1}<k_{2}<\ldots\right)$ converge in $\mathbf{E}$;

(iii) for any sequence of numbers $\varepsilon_{1}, \varepsilon_{2}, \ldots= \pm 1$, the series $\sum \varepsilon_{k} X_{k}$ converges in $\mathbf{E}$.

If $\mathbf{E}$ is a Banach space (say, of random variables with values in $\mathbf{F}$, e.g., $\mathbf{E}=$ $\mathcal{L}^{p}(\mathbf{F}), p \geqslant 1$ ), then, additionally, (i), (ii) and (iii) are equivalent to the condition in $\mathbf{E}$.

(iv) for any bounded sequence $\lambda_{1}, \lambda_{2}, \ldots \in \mathbb{R}$, the series $\sum \lambda_{k} X_{k}$ converges

Although, for a general metric linear space $\mathbf{E}$, (i) does not necessarily imply (iv), in the special case $\mathbf{E}=\mathcal{L}^{0}(\Omega, \mathcal{F}, P ; \mathbf{F})=\mathcal{L}^{0}(\mathbf{F})$ of the space of all random variables with values in a Banach space $\mathbf{F}$, equipped with convergence in probability, we still have the equivalence (i) $\Leftrightarrow$ (ii) $\Leftrightarrow$ (iii) $\Leftrightarrow$ (iv) which is implied by the following

THEOREM. Let $\left(X_{k}\right)$ be a sequence of elements of $\mathcal{L}^{0}(\mathbf{F})$, where $\mathbf{F}$ is a Banach space. Then the series $\sum X_{k}$ converges unconditionally in $\mathcal{L}^{0}(\mathbf{E})$ if, and only if, for each bounded sequence $\lambda_{1}, \lambda_{2}, \ldots \in \mathbb{R}$, the series $\sum \lambda_{k} X_{k}$ converges in $\mathcal{L}^{0}(\mathbf{F})$.

The proof of the above theorem depends crucially on the following elegant lemma, with striking universal constants, concerning generalized Bernoulli random variables.

LEMMA. Let $\lambda=\left(\lambda_{1}, \lambda_{2}, \ldots\right)$ be a sequence of real numbers such that $\left|\lambda_{k}\right|$ $\leqslant 1$, and let $\varpi_{\lambda}$ be a generalized Bernoulli probability distribution on $\{-1,1\}^{\mathrm{N}}$ such that random variables $\{-1,1\}^{\mathbf{N}} \ni\left(\varepsilon_{k}\right) \mapsto \varepsilon_{j}= \pm 1, j=1,2, \ldots$, are independent and such that $\int_{\{-1,1\}^{\mathrm{N}}} \varepsilon_{j} d \varpi_{\lambda}=\lambda_{j}$. Then, for any sequence $x_{1}, x_{2}, \ldots$ of elements of a Banach space $\mathbf{F}$ and for any $n \in \mathcal{N}$,

$$
\varpi\left\{\left(\varepsilon_{k}\right):\left\|\sum_{k=1}^{n} \varepsilon_{k} x_{k}\right\| \geqslant \frac{1}{8}\left\|\sum_{k=1}^{n} \lambda_{k} x_{k}\right\|\right\} \geqslant \frac{1}{8},
$$

where $\varpi=\frac{1}{2}\left(\varpi_{0}+\varpi_{\lambda}\right)$.

Notice that the last inequality implies that, for each $c>0$,

$$
\begin{aligned}
\left\{c: \frac{1}{c} \max _{\varepsilon_{k}= \pm 1} P\left\{\omega:\left\|\sum_{k=1}^{n} \varepsilon_{k} X_{k}(\omega)\right\|>c\right\} \leqslant 1\right\} \\
\subset\left\{\frac{1}{8} c: \frac{1}{c} P\left\{\omega:\left\|\sum_{k=1}^{n} \lambda_{k} X_{k}(\omega)\right\|>c\right\} \leqslant 1\right\},
\end{aligned}
$$


and that the above inclusion, and the definition of the quasi-norm

$$
\|X\|_{\bullet}:=\min \left\{c: c^{-1} P\{\omega:\|X(\omega)\|>c\} \leqslant 1\right\},
$$

on $\mathcal{L}^{0}(\Omega, \mathcal{F}, P ; \mathbf{F})$, give us also the following surprising quantitative result:

TheOREM. Let $X_{1}, X_{2}, \ldots \in \mathcal{L}^{0}(\mathbf{F})$ and let $\lambda_{1}, \lambda_{2}, \ldots \in \mathbb{R}$ be such that $\left|\lambda_{k}\right| \leqslant 1$ for $k=1,2, \ldots$ Then, for each $n \in \mathbb{N}$,

$$
\left\|\sum_{k=1}^{n} \lambda_{k} X_{k}\right\|_{\bullet} \leqslant 8 \max _{\varepsilon_{k}= \pm 1}\left\|\sum_{k=1}^{n} \varepsilon_{k} X_{k}\right\|_{\bullet}
$$

The problem of almost sure convergence in the above setting has also been studied in [23]. We shall say that the series of random variables $\sum_{k=1}^{\infty} X_{k}$ converges unconditionally a.s. if each permutation of $\left(X_{k}\right)$ gives rise to an a.s. convergent series.

For such a (non-metric!!!) convergence, the situation is quite different than the one described at the beginning of this subsection. First of all, it turns out that the a.s. convergence of all the series $\sum_{k=1}^{\infty} \varepsilon_{k} X_{k}$, where $\varepsilon_{k}= \pm 1$ for $k=1,2, \ldots$, is not sufficient for the unconditional a.s. convergence of the series $\sum X_{k}$ (even for real random variables $X_{k}$; see comments below). However, we still have the following

THEOREM. If $\left(X_{k}\right) \subset \mathcal{L}^{0}(\Omega, \mathcal{F}, P ; \mathbf{F})$ and if the series $\sum X_{k}(\omega)$ converges unconditionally a.s., then, for each bounded sequence $\left(\lambda_{k}\right) \subset \mathbb{R}$, the series $\sum \lambda_{k} X_{k}(\omega)$ converges unconditionally a.s.

The proof follows directly from the following propositions and lemma. The first one can be labeled as a kind of the 'uniformity-of-the-a.s.-divergence' result.

Proposition. If the series $\sum X_{k}(\omega)$ unconditionally converges a.s., then, for each sequence $\varepsilon_{1}, \varepsilon_{2}, \ldots= \pm 1$, the series $\sum \varepsilon_{k} X_{k}(\omega)$ converges unconditionally a.s.

Proposition. If the series $\sum \varepsilon_{k} X_{k}(\omega)$ converges a.s. for each $\varepsilon_{1}, \varepsilon_{2}, \ldots$ $= \pm 1$, then the series $\sum \lambda_{k} X_{k}(\omega)$ converges a.s. for each bounded sequence $\lambda_{1}, \lambda_{2}, \ldots \in \mathbb{R}$.

LEMmA. Let $\lambda_{1}, \lambda_{2}, \ldots \in \mathbb{R}$ be a sequence such that $\left|\lambda_{k}\right| \leqslant 1$ for $k=$ $1,2, \ldots$, and let $x_{1}, x_{2}, \ldots$ be a sequence of elements of a Banach space $\mathbf{F}$. Then

$$
\varpi\left\{\left(\varepsilon_{i}\right): \lim _{n \rightarrow \infty} \sup _{n \leqslant p \leqslant q}\left\|\sum_{k=p}^{q} \varepsilon_{k} x_{k}\right\| \geqslant \frac{1}{8} \lim _{n \rightarrow \infty} \sup _{n \leqslant p \leqslant q}\left\|\sum_{k=p}^{q} \lambda_{k} x_{k}\right\|\right\} \geqslant \frac{1}{8},
$$

with $\varpi$ as defined above. 
The above results have been employed in a variety of applications. As an example we cite [39] where the authors used what they call the Ryll-NardzewskiWoyczyński theorem to establish the uniform weak law for tail series of weighted sums of random elements in Banach spaces.

\section{ERGODIC THEORY}

Ryll-Nardzewski's discoveries of ergodic behavior in different contexts have been widely used and included in the canon of probability theory for a long time. In Chapter IX of his 1955 celebrated monograph [5]] Michel Loève extensively used CRN's, and his collaborators' results contained in [2], [3], and [9]. And Patrick Billingsley in his 1965 book on ergodic theory and information [30] provides the detailed exposition of CRN's ergodic theorem for continued fractions. Also, in his often used probability textbook [32] (p. 128), Leo Breiman writes: "A good development of point processes and proofs of more general versions of [ergodic theorem for point processes] and the ergodic property of the recurrence times is in Ryll-Nardzewski [20]." A more recent book by Richard Durrett [36] includes CRN's ergodic theorems for continued fractions, and Ulrich Krengel's comprehensive monograph [49] on ergodic theorems also contains a coverage of CRN's work in the area. Throughout the remainder of the section we will briefly summarize CRN's work on ergodic theory contained in [2], [3].

Consider a set $\mathcal{X}$ with a $\sigma$-finite measure $\mu$ defined on a $\sigma$-field $\mathcal{M}$ of subsets of $\mathcal{X}$. By $\varphi: \mathcal{X} \rightarrow \mathcal{X}$ we will denote a transformation such that $\varphi^{-1} E \in \mathcal{M}$ for $E \in \mathcal{M}$, and $\mu\left(\varphi^{-1} E\right)=0$ if $\mu(E)=0$.

The classical individual and mean ergodic theorems of Birkhoff and von Neumann state that if $\varphi$ is measure preserving (that is, $\mu\left(\varphi^{-1} E\right)=\mu(E)$ ), then, for each $f \in L_{1}(\mathcal{X}, \mathcal{M}, \mu)$, there exists a $g \in L_{1}(\mathcal{X}, \mathcal{M}, \mu)$ such that, as $n \rightarrow \infty$,

$$
\frac{1}{n} \sum_{i=0}^{n-1} f\left(\varphi^{i}(x)\right) \rightarrow g(x),
$$

on a set of full $\mu$-measure and in $L_{1}(\mu)$ (the latter holds under the extra condition that the measure $\mu$ is finite).

In [2], CRN has demonstrated the following result:

THEOREM. The statement of Birkhoff's individual ergodic theorem is equivalent to each of the following three conditions:

(i) For each set $A$ and each set $Y$ with $\mu(Y)<\infty$,

$$
\lim _{n} M_{n}(A, Y) \leqslant K \mu(A),
$$

where $K$ is a constant number, and

$$
M_{n}(A, Y):=\frac{1}{n} \sum_{k=0}^{n-1} \mu\left(Y \cap \varphi^{-k} A\right) .
$$


(ii) For each set $A$ and each set $Y$ with $\mu(Y)<\infty$,

$$
\limsup _{n} M_{n}(A, Y) \leqslant K \mu(A) .
$$

(iii) There is an ascending sequence $\left\{Y_{j}\right\}$ of sets such that $X=\bigcup_{j} Y_{j}$, and the condition

$$
M_{n}(A, Y):=\frac{1}{n} \sum_{k=0}^{n-1} \mu\left(Y \cap \varphi^{-k} A\right)
$$

holds for $Y=Y_{j}, j=1,2, \ldots$, and each set $A$.

In the context of the mean ergodic theorem he proves

THEOREM. The statement of von Neumann's mean ergodic theorem is equivalent to the following condition:

$$
\frac{1}{n} \sum_{k=0}^{n-1} \mu\left(\varphi^{-k} E\right) \leqslant K \mu(E)
$$

for each $E \in \mathcal{M}$ and $n=1,2, \ldots$

On the following pages of Volume 12 of Studia Mathematica (see [3]) CRN addresses the then novel problem of ergodic behavior of the transformation induced by continued fractions which was suggested by Edward Marczewski.

Denoting by $\mathcal{X}$ the set of irrational numbers,

$$
x=\frac{1 \mid}{\mid c_{1}}+\frac{1 \mid}{\mid c_{2}}+\frac{1 \mid}{\mid c_{3}}+\ldots,
$$

in the interval $(0,1)$, Marczewski defined the transformation

$$
\delta(x):=\frac{1}{x}-\left\lfloor\frac{1}{x}\right\rfloor=\frac{1 \mid}{\mid c_{2}}+\frac{1 \mid}{\mid c_{3}}+\frac{1 \mid}{\mid c_{4}}+\ldots
$$

The transformation is indecomposable with respect to the Lebesgue measure, that is, all the invariant sets are either of measure zero or one, but it does not preserve the Lebesgue measure. This nontrivial fact was stated in Theorem 1 of [3], which was attributed to Knopp [48]. However, CRN's proof was new. Surprisingly, RyllNardzewski found a measure invariant under $\delta$, and proved the following ergodic theorem for it:

THEOREM. The measure

$$
\nu(E)=\frac{1}{\log 2} \int_{E} \frac{d x}{1+x},
$$

defined on all Lebesgue measurable subsets of $\mathcal{X}$, is invariant under the transformation $\delta$, and the classes of all sets of measure zero, and that of integrable 
functions, are the same for $\nu$ and for the Lebesgue measure. Moreover, for each Lebesgue integrable function $f$,

$$
\frac{1}{n}\left[f(x)+f(\delta(x))+\ldots+f\left(\delta^{n-1}(x)\right)\right] \rightarrow \frac{1}{\log 2} \int_{0}^{1} f(x) \frac{d x}{1+x},
$$

almost everywhere.

\section{DE FINETTI'S EXCHANGEABLE SEQUENCES, SELECTORS, AND CONDITIONAL DISTRIBUTIONS}

In this final section we describe a few CRN's results which, while not being the constitutive part of several of his comprehensive theories, had a lasting influence in several areas of mathematics and its applications.

5.1. De Finetti's exchangeable sequences. The definition of exchangeable sequences in the sense of de Finetti has been recalled in Subsection 2.1. In the 1957 paper [119], CRN made a thorough study of different notions of symmetry, which turn out to be equivalent to the notion of exchangeability.

He started out by considering the product space $\boldsymbol{X}=\mathbb{R}^{\infty}$ of sequences $\boldsymbol{x}=$ $\left(x_{1}, x_{2}, \ldots\right)$, with $\mathcal{B}$ denoting the $\sigma$-field of Borel subsets of $\boldsymbol{X}$, and $\mathcal{M}$ standing for the class of probability measures on $(\boldsymbol{X}, \mathcal{B})$.

In this setting $\xi(\boldsymbol{x})=x_{n}$ is a random variable and, for $\mu \in \mathcal{M}$,

$$
F_{k}\left(t_{1}, \ldots, t_{k}\right)=\mu\left(x_{1}<t_{1}, \ldots, x_{k}<t_{k}\right)
$$

is the $k$-dimensional cumulative distribution function. Introducing the shift operator $\phi(\boldsymbol{x}):=\left(x_{2}, x_{3}, \ldots\right)$, one says that $\mu \in \mathcal{M}$ is stationary if

$$
\mu(E)=\mu\left(\phi^{-1} E\right)
$$

for any $E \in \mathcal{B}$ (we write then $\mu \in \mathcal{M}_{\mathrm{st}}$ ). A set $B$ is invariant (symbolically, $B \in$ $\mathcal{B}_{\text {inv }}$ ) if $B=\phi^{-1} B$. A measure $\mu$ is indecomposable, in brief, $\mu \in \mathcal{M}_{\text {ind }}$ (ergodic, in the contemporary terminology) if $\mu(E)=0$ or 1 for all $E \in \mathcal{B}_{\text {inv }}$.

The following three different concepts of symmetry were considered in [19]:

(sym) A measure $\mu \in \mathcal{M}$ is symmetric $\left(\mu \in \mathcal{M}_{\text {sym }}\right.$ ) if, for every increasing sequence of integers $n_{1}<n_{2}<\ldots$, and any $\mu$-integrable function $f$,

$$
\int f\left(x_{1}, x_{2}, \ldots\right) d \mu=\int f\left(x_{n_{1}}, x_{n_{2}}, \ldots\right) d \mu .
$$

(ssym) A measure $\mu \in \mathcal{M}$ is strongly symmetric $\left(\mu \in \mathcal{M}_{\text {ssym }}\right.$ ) if, for every sequence of (not necessarily increasing!) different positive integers $n_{1}, n_{2}, \ldots$, and any $\mu$-integrable function $f$,

$$
\int f\left(x_{1}, x_{2}, \ldots\right) d \mu=\int f\left(x_{n_{1}}, x_{n_{2}}, \ldots\right) d \mu .
$$


(prod) $\mu$ is a product measure $\left(\mu \in \mathcal{M}_{\mathrm{pr}}\right.$ ) if it is stationary and

$$
\left.\int f_{1}\left(x_{1}\right) f_{2}\left(x_{2}\right), \ldots\right) f_{k}\left(x_{k}\right) d \mu=\int f_{1}\left(x_{1}\right) d \mu \int f_{2}\left(x_{2}\right) d \mu \ldots \int f_{k}\left(x_{k}\right) d \mu
$$

for all $k=1,2, \ldots$ and all sequences of $\mu$-integrable functions $f_{j}, j=1,2, \ldots$

Product measures are indecomposable. They correspond to sequences of independent and identically distributed random variables, and Lemma 1 in [19] shows that every product measure is symmetric in the strong sense. Then Theorem 2 in [19] proves that every symmetric and indecomposable measure is a product measure.

Finally, the paper introduces the concept of conditional measure $\mu\left(E \mid \mathcal{B}_{\text {inv }}\right)$ with respect to $\mathcal{B}_{\text {inv }}$, defines the regular conditional probability $\mu(E \mid \boldsymbol{x})$, and establishes the following facts: if $\mu \in \mathcal{M}_{\text {sym }}$, then $\mu(\cdot \mid x) \in \mathcal{M}_{\text {sym }}$; if $\mu \in \mathcal{M}_{\text {st }}$, then $\mu(\cdot \mid \boldsymbol{x}) \in \mathcal{M}_{\text {st }}$; and if $\mu \in \mathcal{M}_{\text {st }}$, then $\mu(\cdot \mid \boldsymbol{x}) \in \mathcal{M}_{\text {ind }}$. The main result of the paper is the following theorem.

THEOREM. Suppose that $\mu \in \mathcal{M}_{\mathrm{sym}}$, and let $\mu(E \mid \boldsymbol{x})$ denote the conditional measure with respect $\mathcal{B}_{\text {inv }}$. Then,

(a) $\mu(\cdot \mid \boldsymbol{x})$ is a product measure $\mu$-a.e.;

(b) $\mu(E)=\int \mu(E \mid \boldsymbol{x}) d \mu, E \in \mathcal{B}$;

(c) $\mu$ is symmetric in the strong sense.

The ideas developed in [19], and [20], had a broad influence on more recent literature on the subject and, for example, were of crucial importance in Chapter 11 of the monograph on probability theory written by Olav Kallenberg, [43].

5.2. Conditional distributions. In 1963 , CRN returned to the fundamental problems related to conditional distributions in a joint paper with a Berkeley mathematician, David Blackwell. The set-up was as follows: Let $\Omega$ be a Borel subset of a complete separable metric space, $\mathcal{B}$ the class of Borel subsets of $\Omega$, and $P$ a probability measure on $\Omega$. Let $f$ be a real-valued random variable on $\Omega$, and $\mathcal{A}$ be the $\sigma$-field generated by $f$. In standard probability textbooks (see, e.g., Durrett [36]) one can find theorems demonstrating the existence of regular conditional probabilities $Q(\omega, B)$ given $f$, satisfying the following conditions:

- For each $\omega \in \Omega, Q(\omega, \cdot)$ is a probability measure on $\mathcal{B}$.

- For each $B \in \mathcal{B}, Q(\omega, B)$ is $\mathcal{A}$-measurable.

- For every $A \in \mathcal{A}, B \in \mathcal{B}$,

$$
\int_{A} Q(\omega, B) P(d \omega)=P(A \cap B) .
$$

A conditional distribution $Q$ is proper if $Q(\omega, A)=1$ for $\omega \in A \in \mathcal{A}$. It is well known that conditional distribution, proper for almost all points of $\Omega$, i.e. outside of an exceptional set $N$, always exists. Blackwell and Ryll-Nardzewski [21] demonstrated that, in general, the exceptional set cannot be removed. In particular, they observed that this is a case if the range of $f$ is not Borel. 
5.3. The theorem on selectors. One of the most frequently cited results of CRN (according to the Mathematical Reviews) is the so-called Kuratowski-RyllNardzewski's theorem on selectors proven in [22]. Although this theorem is not, strictly speaking, a probabilistic result, it has found diverse applications in statistics, stochastic geometry, stochastic games, stochastic control, and also in economics, deterministic and stochastic dynamic optimization, and other areas (see, e.g., [38]). This theorem is fundamental in the theory of multivalued functions, random fixed point theorems for a multivalued contraction mappings, and the theory of martingales in Banach spaces. It is basic in stochastic geometry as it allows to define, for example, the expectation of a random set. An unexpected application was found in the proof of an extension of the Choquet-Bishop-de Leeuw theorem due to Gerry Edgar. Diestel and Uhl, describing his result in [35] (p. 145) write "the proof is a beautiful mixture of martingale methods, ... a selection theorem of Kuratowski and Ryll-Nardzewski."

The Kuratowski-Ryll-Nardzewski theorem about selectors considers two sets $X, Y$, and a set-valued function $F(x) \subset Y$ defined for each $x \in X$. A selector is defined as any element of the Cartesian product $\prod_{x \in X} F(x)$. Now, consider a field $\mathcal{L}$ of subsets of $X$, and a countable additive family $\mathcal{S}$ generated by $\mathcal{L}$.

THEOREM. Let $Y$ be a complete separable metric space, and let $F: X \rightarrow 2^{Y}$ be such that $\{x: F(x) \cap G \neq \emptyset\} \in \mathcal{S}$, whenever $G \subset Y$ is open. Then there is a selector $f: X \rightarrow Y$ such that $f^{-1}(G) \in \mathcal{S}$, whenever $G \subset Y$ is open.

Assuming that $\mathcal{L}$ is a countable additive field of subsets of $X$, a selector $f$ : $X \rightarrow Y$ is said to be $\mathcal{L}$-measurable if, for any open $G \subset Y$, we have $f^{-1}(G) \in \mathcal{L}$. In this context Kuratowski and CRN also proved the following result:

THEOREM. Let $Y$ be a complete separable metric space, and let $F: X \rightarrow 2^{Y}$ be $\mathcal{L}$-measurable. Then an $\mathcal{L}$-measurable selector exists.

Acknowledgments. The authors thank Stanisław Kwapień for his suggestions concerning the description of the Kuratowski-Ryll-Nardzewski theorem and, in particular, for reference [35]. Jan Rosiński carefully reviewed the first draft of this article and we appreciate his help in improving our presentation.

Note. The references listed below start with selected CRN's papers in probability theory (in chronological order), and are followed by other relevant citations (in alphabetical order).

\section{REFERENCES}

[1] J. Mikusiński and C. Ryll-Nardzewski, Sur le produit de composition, Studia Math. 12 (1951), pp. 51-57.

[2] C. Ryll-Nardzewski, On the ergodic theorems. I (Generalized ergodic theorems), Studia Math. 12 (1951), pp. 65-73. 
[3] C. Ryll-Nardzewski, On the ergodic theorems. II (Ergodic theory of continued fractions), Studia Math. 12 (1951), pp. 74-79.

[4] C. Ryll-Nardzewski and H. Steinhaus, Sur les fonctions indépendantes. IX (Séries des fonctions positives), Studia Math. 12 (1951), pp. 102-107.

[5] C. Ryll-Nardzewski, Sur les suites et les fonctions également réparties, Studia Math. 12 (1951), pp. 143-144.

[6] C. Ryll-Nardzewski and H. Steinhaus, Sur les séries de Taylor, Studia Math. 12 (1951), pp. 159-165.

[7] J. Mikusiński and C. Ryll-Nardzewski, Sur l'opérateur de translation, Studia Math. 12 (1951), pp. 205-207.

[8] C. Ryll-Nardzewski, Certaines théorèmes des moments, Studia Math. 12 (1951), pp. 225 226.

[9] S. Hartman, E. Marczewski, and C. Ryll-Nardzewski, Théorèmes ergodiques et leurs applications, Colloq. Math. 2 (1951), pp. 109-123.

[10] C. Ryll-Nardzewski, D. Blackwell's conjecture on power series with random coefficients, Studia Math. 13 (1953), pp. 30-36.

[11] C. Ryll-Nardzewski, Sur la convergence des séries de puissances de l'opérateur différentiel, Studia Math. 13 (1953), pp. 37-40.

[12] C. Ryll-Nardzewski, Sur les séries de puissances dans le calcul opératoire, Studia Math. 13 (1953), pp. 41-47.

[13] J. Mikusiński and C. Ryll-Nardzewski, A theorem on bounded moments, Studia Math 13 (1953), pp. 51-55.

[14] J. Mikusiński and C. Ryll-Nardzewski, Un théorème sur le produit de composition des fonctions de plusieurs variables, Studia Math. 13 (1953), pp. 62-68.

[15] K. Florek, E. Marczewski, and C. Ryll-Nardzewski, Remarks on the Poisson stochastic process. I, Studia Math. 13 (1953), pp. 122-129.

[16] S. Hartman and C. Ryll-Nardzewski, Théorèmes abstraits de Kronecker et les fonctions presque périodiques, Studia Math. 13 (1953), pp. 296-311.

[17] C. Ryll-Nardzewski, On the non-homogeneous Poisson process. I, Studia Math. 14 (1954), pp. $124-128$.

[18] C. Ryll-Nardzewski, Remarks on the Poisson stochastic process. III (On a property of the homogeneous Poisson process), Studia Math. 14 (1954), pp. 314-318.

[19] C. Ryll-Nardzewski, On stationary sequences of random variables and the de Finetti's equivalence, Colloq. Math. 4 (1957), pp. 149-156.

[20] C. Ryll-Nardzew ski, Remarks on processes of calls, in: Proceedings of the Fourth Berkeley Symposium on Mathematical Statistics and Probability. Volume 2: Contributions to Probability Theory, Univ. California Press, Berkeley, CA, 1961, pp. 455-465.

[21] D. Blackwell and C. Ryll-Nardzewski, Non-existence of everywhere proper conditional distributions, Ann. Math. Statist. 34 (1963), pp. 223-225.

[22] K. Kuratowski and C. Ryll-Nardzewski, A general theorem on selectors, Bull. Acad. Polon. Sci. Sér. Sci. Math. Astronom. Phys. 13 (1965), pp. 397-403.

[23] K. Musiał, C. Ryll-Nardzewski, and W. A. Woyczyński, Convergence presque sûre des séries aléatoires vectorielles à multiplicateurs bornés, C. R. Acad. Sci. Paris Sér. A 279 (1974), pp. 225-228.

[24] C. Ryll-Nardzewski and W. A. Woyczyński, Convergence en mesure des séries aléatoires vectorielles à multiplicateur borné, in: Séminaire Maurey-Schwartz (1973-1974): Espaces $L^{p}$, applications radonifiantes et géométrie des espaces de Banach. Annexe, Centre de mathématiques, École polytechnique, Paris 1974.

[25] C. Ryll-Nardzewski and W. A. Woyczyński, Bounded multiplier convergence in measure of random vector series, Proc. Amer. Math. Soc. 53 (1975), pp. 96-98.

[26] J. Rosiński and C. Ryll-Nardzewski, Cylindrical measures on topological groups, Probab. Math. Statist. 6 (1985), pp. 167-172. 
[27] T. Rolski and C. Ryll-Nardzewski, Is the dying individual the oldest?, Stochastic Process. Appl. 24 (1987), pp. 133-142.

\section{Other references (in alphabetical order)}

[28] F. B accelli and B. Błaszczyszyn, Stochastic Geometry and Wireless Networks. Volume I: Theory, Foundation and Trends in Networking, vol. 3, no. 3-4, Now Publishers, 2009.

[29] P. Biler, P. Krupski, G. Plebanek, and W. A. Woyczyński, Lwów of the West: A brief history of Wroctaw's New Scottish Book, in: The Scottish Book: Mathematics from the Scottish Café, R. Mauldin (Ed.), second edition, Springer-Birkhäuser 2016, pp. 291-296.

[30] P. Billingsley, Ergodic Theory and Information, Wiley, New York-London-Sydney 1965.

[31] É. Borel, Sur les séries de Taylor, C. R. Acad. Sci. Paris 123 (1896), pp. 1051-1052.

[32] L. Breiman, Probability, Addison-Wesley, Reading, MA, 1968.

[33] H. Cramér, A contribution to the theory of stochastic processes, in: Proceedings of the Second Berkeley Symposium on Mathematical Statistics and Probability, Univ. California Press, Berkeley, CA, 1951, pp. 329-340.

[34] D. J. Daley and D. Vere-Jones, An Introduction to the Theory of Point Processes, Volume I: Elementary Theory and Methods, Springer, 2003.

[35] J. Diestel and J. J. Uh1, Jr., Vector Measures, AMS, Providence, R.I., 1977.

[36] D. Durrett, Probability: Theory and Examples, fourth edition, Cambridge Univ. Press, 2010.

[37] W. Feller, An Introduction to Probability Theory and Its Applications, vol. 2, second edition, Wiley, New York 1971.

[38] K. Hinderer, U. Riedel, and M. Stieglitz, Dynamic Optimization: Deterministic and Stochastic Models, Springer, 2016.

[39] T. C. Hu, E. Nam, A. Rosalsky, and A. I. Volodin, An application of the RyllNardzewski-Woyczyński theorem to a uniform weak law for tail series of weighted sums of random elements in Banach spaces, Statist. Probab. Lett. 48 (2000), pp. 369-374.

[40] J.-P. Kahane, Sur les fonctions sommes de séries trigonométriques absolument convergentes, C. R. Acad. Sci. Paris 240 (1955), pp. 36-37.

[41] J.-P. Kahane, Some Random Series of Functions, Heath and Co, 1968.

[42] O. Kallenberg, Random Measures, Akademie-Verlag-Academic Press, Berlin 1983.

[43] O. Kallenberg, Foundations of Modern Probability, Springer, 2002.

[44] A. Ya. Khinchin, Mathematical Methods of the Theory of Mass Service, Tr. Mat. Inst. Steklova, vol. 49, Moscow 1955.

[45] A. Ya. Khinchin, Flows of random events without after-effects, Teor. Verojatnost. i Primenen. 1 (1956), pp. 3-17.

[46] J. F. C. King man, Completely random measures, Pacific J. Math. 21 (1967), pp. 59-78.

[47] J. F. C. Kingman, Poisson Processes, Clarendon Press, Oxford 1993.

[48] K. Knopp, Mengentheoretische Behandlung einiger Probleme der diophantischen Approximationen und der transfiniten Wahrscheinlichkeiten, Mathematische Annalen 95 (1926), pp. 409-426.

[49] U. Krengel, Ergodic Theorems, W. de Gruyter, Berlin-New York 1985.

[50] S. Kwapień and W. A. Woy czyński, Random Series and Stochastic Integrals: Single and Multiple, Birkhäuser-Boston, 1992.

[51] M. Loève, Probability Theory: Foundations, Random Sequences, Van Nostrand, New York 1955.

[52] E. Marczewski, Remarks on the Poisson stochastic process (II), Studia Math. 13 (1953), pp. 130-136.

[53] A. Prékopa, On secondary processes generated by a random point distribution of Poisson type, Ann. Univ. Sci. Budapest 1 (1958), pp. 153-170.

[54] A. Rényi, Remarks on the Poisson process, Studia Sci. Math. Hungar. 2 (1967), pp. 119-123. 
[55] T. Rolski, H. Schmidli, V. Schmidt, and J. Teugels, Stochastic Processes for Insurance and Finance, Wiley, Chichester 1999.

[56] H. Stein hau s, Über die Wahrscheinlichkeit dafür, dass der Konvergenzkreis einer Potenzreihe ihre natürliche Grenze ist, Math. Z. 31 (1930), pp. 408-416.

[57] D. Szász and W. A. Woyczyński, Poissonian random measures and linear processes with independent pieces, Bull. Acad. Polon. Sci. Sér. Sci. Math. Astronom. Phys. 18 (1970), pp. 475-482.

Tomasz Rolski

Mathematical Institute

University of Wrocław

pl. Grunwaldzki $2 / 4$

50-384 Wrocław, Poland

E-mail: tomasz.rolski@gmail.com
Wojbor A. Woyczyński

Department of Mathematics,

Applied Mathematics and Statistics Center for Stochastic and Chaotic Processes

in Sciences and Technology

Case Western Reserve University

Cleveland, OH 44106

E-mail: wojbor.woyczynski@case.edu

Received on 12.3.2015;

revised version on 26.6.2016 